\title{
Diastolic Dysfunction in Patients with Chronic Obstructive Pulmonary Disease: A Meta-Analysis of Case Controlled Studies
}

Angelina Zhyvotovska, Denis Yusupov, Haroon Kamran, Tarik Al-Bermani, Rishard Abdul, Samir Kumar, Nikita Mogar, Angeleque Hartt, Louis Salciccioli, and Samy I. McFarlane*

Department of Medicine, Division of Endocrinology, State University of New York-Downstate Medical Center, Brooklyn, New York

\section{Abstract}

Background: Chronic obstructive pulmonary disease (COPD) and left ventricular diastolic dysfunction (LVDD) are major causes of morbidity and mortality and have overlapping symptomatology including cough and dyspnea. Whether COPD is a risk factor for LVDD remains largely unclear.

The objective of this meta-analysis was to determine if the prevalence of the LVDD as determined by echocardiographic parameters is increased in COPD patients.

Methods: We used a time-and-language-restricted search strategy resulting in identification of 4,912 studies of which 15 studies met our apriori inclusion criteria; 4,897 were excluded, such duplicates, foreign language articles were excluded. We performed a meta-analysis of standard echo parameters on the fifteen case control studies related to diastolic dysfunction. The meta-analysis was performed using Review Manager, version 5.3 (Cochrane Collaboration).

Results: A total of 15 studies with 1,403 subjects were included. There were no differences in left ventricular ejection fraction between COPD and non-COPD population. Patients with COPD had prolonged isovolumetric relaxation time (IVRT) (mean difference 20.84 [95\% CI 12.21, 29.47]; $\mathrm{P}<0.00001$ ), lower E/A ratio (mean difference - 0.24 [95\% CI -0.34, 00.14]; $\mathrm{P}<0.00001$ ), higher transmitral A wave peak velocity (Apv) (mean difference 11.71 [95\% CI 4.80, 18.62]; $\mathrm{P}<0.00001$ ), higher E/e' ratio (mean difference 1.88 [95\% CI 1.23, 2.53]; $\mathrm{P}<0.00001$ ), lower mitral E wave peak velocity (Epv) (mean difference -8.74 [95\% CI -13.63, -3.85]; $\mathrm{P}<0.0005$ ), prolonged deceleration time (DT) (mean difference 50.24 [95\% CI $15.60,84,89] ; \mathrm{P}<0.004)$, a higher right ventricular end diastolic diameter (RVEDD) (mean difference 8.02 [95\% CI 3.45, 12.60]; $\mathrm{P}<0.0006)$ compared to controls. COPD patients had a higher pulmonary arterial pressure (mean difference 10.52 [95\% CI 3.98, 17.05]; $\mathrm{P}<0.002$ ). Differences in septal e' velocity (mean difference -2.69 [95\% CI -6.07, 0.69]; $\mathrm{P}<0.12$ ) and in lateral e' velocity (mean difference -2.84 [95\% CI 5.91, 0.24]; $\mathrm{P}<0.07)$ trended towards significance but did not meet our cutoff for statistical significance $(\mathrm{p}<0.05)$

Conclusions: Patients with COPD are more likely to have LVDD as established by echocardiographic parameters. Our findings are likely explainable, in part, by factors such as lung hyperinflation, chronic hypoxia, hypercapnia, systemic inflammation, increased arterial stiffness, subendocardial ischemia, as well as ventricular interdependence; all of which might contribute to the pathogenesis of diastolic dysfunction. Further research is needed to elucidate the pathophysiologic mechanisms of increased LVDD in the COPD population with the potential impact on developing effective therapeutic interventions for these serious disorders.

\section{Introduction}

Chronic obstructive pulmonary disease (COPD) is an important cause of morbidity and mortality around the world. Estimates show that in 2030 COPD will become the third leading cause of death worldwide [1]. Comorbidities in COPD have been studied extensively as COPD patients frequently suffer from comorbidities such as cardiovascular and cerebrovascular disease, lung cancer and diabetes. Research studying the impact of multimorbidities facing COPD patients is necessary as it drives the development of better intervention and diagnostic strategies. Comorbidities have a significant impact on mortality, termed by Divo et al. as the "comorbidome" [1]. There is evidence that comorbidities increase the risk for exacerbations, reduce health status, and increase the risk of mortality $[3,4]$.

The diagnosis of heart failure with preserved ejection fraction (HFpEF) is more challenging than the diagnosis of heart failure with reduced ejection fraction (HFrEF) [5]. According to European Society of Cardiology, patients with HFpEF have an increase in LV wall thickness that results increased filling pressures. The precursor to HFpEF is abnormal left ventricular relaxation, termed left ventricular 270-4488; E-mail: smcfarlane@downstate.edu original author and source are credited.

\section{Publication History:}

Received: July 31, 2019

Accepted: September 17, 2019

Published: September 19, 2019

\section{Keywords:}

Diastolic Dysfunction, Chronic Obstructive Pulmonary Disease, Echocardiography, Heart Failure with Preserved Ejection Fraction

LVDD involves functional abnormalities including decreased myocyte relaxation, and decreased filling or distensibility of the LV, regardless of whether the left ventricular ejection fraction (LVEF) is normal or abnormal or whether the patient is symptomatic or not. LVDD has been associated with coronary artery disease (CAD),

"Corresponding Author: Prof. Samy I. McFarlane, Division of Endocrinology, Department of Internal Medicine, State University of New York, Downstate Medical Center, Brooklyn, New York,11203, USA, Tel: 718-270-6707, Fax: 718-

Citation: Zhyvotovska A, Yusupov D, Kamran $\mathrm{H}$, Al-Bermani $\mathrm{T}$, Abdul $\mathrm{R}$, et al. (2019) Diastolic Dysfunction in Patients with Chronic Obstructive Pulmonary Disease: A Meta-Analysis of Case Controlled Studies. Int J Clin Res Trials 4: 137. doi: https://doi.org/10.15344/2456-8007/2019/137

Copyright: (C) 2019 Zhyvotovska et al. This is an open-access article distributed under the terms of the Creative Commons Attribution License, which permits unrestricted use, distribution, and reproduction in any medium, provided the 
Citation: Zhyvotovska A, Yusupov D, Kamran H, Al-Bermani T, Abdul R, et al. (2019) Diastolic Dysfunction in Patients with Chronic Obstructive Pulmonary Disease: A Meta-Analysis of Case Controlled Studies. Int J Clin Res Trials 4: 137. doi: https://doi.org/10.15344/2456-8007/2019/137

Page 2 of 11

Diabetes (DM) and obesity [8], hypertension, and rheumatoid Arthritis (RA) [9]. Consideration for LVDD is an important part in the evaluation of a patient with dyspnea. The echocardiogram is the key diagnostic modality for identifying diagnostic dysfunction. It can also identify concurrent disorders and classify diastolic dysfunction by grades. The importance of identifying LVDD in the COPD population is because it can contribute to dyspnea and lead to heart failure. Both COPD and heart failure exacerbations can present similarly posing a challenge in distinguishing one from the other, even more challenging is that symptoms of one may be incremental to the other.

\section{Methods}

\section{Literature search and data sources}

An electronic search was performed by two (2) investigators using the PUBMED, Medline, and Cochrane Library databases at the library of the University Hospital of Brooklyn. We used a time (from 2000 to 2018) and language restricted (English) search strategy to identify 4912 articles. Five sets of search terms were used to ensure an adequate and comprehensive literature review. These included "Doppler and COPD," "Diastolic and COPD," "Diastolic dysfunction and COPD," "Atrial and COPD," and "Ventricular and COPD." After title and abstract review, the bibliographies of studies that met our inclusion criteria were examined for suitable additional literature. We excluded studies that were not published to maintain a high-quality meta-analysis.

\section{Study selection and quality assessment}

The inclusion was limited to studies that were (1) case-control in design, (2) compared transthoracic echocardiographic (TTE) parameters in COPD patients to non-COPD patients and (3) performed after January 2000. For case control studies that did not include the relevant TTE parameters, the corresponding authors were contacted to provide such data. When no response was attained, the studies were left out of the data pool. No study was excluded based on the size of the study population, but duplicate articles were excluded. For each study, we applied the Newcastle-Ottawa Scale to assess its quality. The quality scale included three components: selection of the study groups with points ranging from 0 to 4 ; compatibility of the study groups, with points from 0 to 2 ; and ascertainment of study groups exposure, points from 0 to 3 . The maximum for each study is 9 , studies with less than 5 points carry the high risk of bias and were excluded $(\mathrm{N}=2)$.

\section{TTE parameters}

In 2016, the ASE/EACVI published an updated guideline for the assessment of LVDD using echocardiography. Their recommendation supports a comprehensive study observing both transmitral inflow parameters and tissue doppler parameters. Various parameters they recommend obtaining include annular e prime velocity: septal e' $<7$ $\mathrm{cm} / \mathrm{sec}$ and/or lateral e' $<10 \mathrm{~cm} / \mathrm{sec}$, average $\mathrm{E} / \mathrm{e}^{\prime}$ ratio $>14$, LA volume index $>34 \mathrm{~mL} / \mathrm{m}^{2}$, and peak TR velocity $>2.8 \mathrm{~m} / \mathrm{sec}$. Many other parameters have diagnostic and prognostic utility [17]. All but one study in our analysis was published prior to these recommendations. For the purpose of this analysis, studies reporting any LVDD parameters were included in this meta-analysis. The TTE parameters that were most commonly used in the studies included isovolumetric relaxation time (IVRT, msec), E/A ratio, transmitral A wave velocity (meters/second), lateral E' velocity (meters/second), E-Wave peak velocity (Epv) (meters/second), and deceleration time (DT, msec). Many of these LVDD parameters change in different directions with increasing diastolic dysfunction grade, highlighting the difficulty in diagnosing LVDD.

\section{Data extraction}

A total of 4,892 studies were excluded for not meeting the inclusion criteria. Eighteen (18) studies meet the inclusion criteria and were reviewed in full; 1 article was removed due to unclear findings, and an additional 2 were removed because the results for their control groups were unclear. Two (2) investigators extracted the data from the fifteen (15) included studies [18-32] with the pooled data represented in tables 1 and 2. Patient characteristics including age, BMI and comorbidity status for each cohort of the individual studies were extracted (Table 3), as well as TTE parameters. The Newcastle-Ottawa Scale for case-control studies was used to assess the quality of the selected articles.

\section{Statistical analysis}

The meta-analysis was performed using Review Manager, version 5.3 (Cochrane Collaboration). Mean differences were evaluated along with 95\% confidence intervals (95\% CIs). Each included echocardiographic parameter was summarized using the randomeffects model. Heterogeneity, Cochran's Q, tau-squared test, and $\mathrm{I}^{2}$ index were assessed for each study.

\section{Results}

There were 1,403 subjects, of which 706 had COPD. In all studies, the diagnosis of COPD was made according to the guidelines of the Global Initiative for Chronic Obstructive Lung Disease (GOLD) criteria.

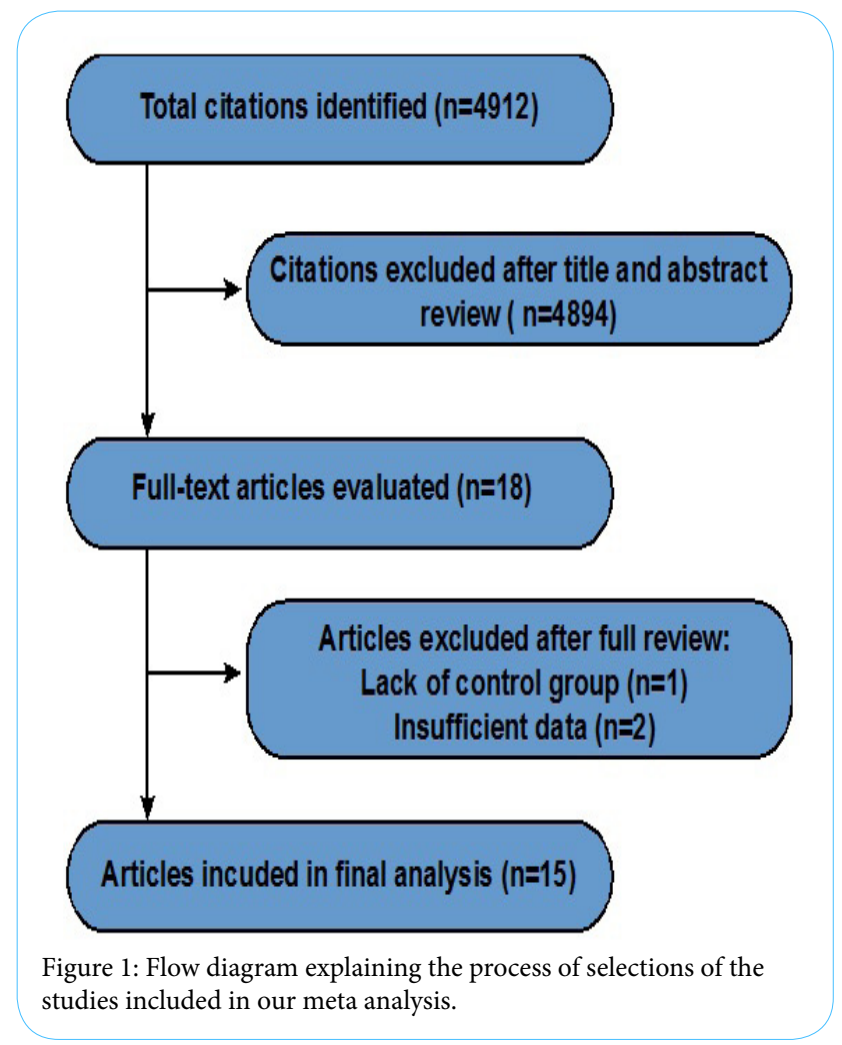


Citation: Zhyvotovska A, Yusupov D, Kamran H, Al-Bermani T, Abdul R, et al. (2019) Diastolic Dysfunction in Patients with Chronic Obstructive Pulmonary Disease: A Meta-Analysis of Case Controlled Studies. Int J Clin Res Trials 4: 137. doi: https://doi.org/10.15344/2456-8007/2019/137

Page 3 of 11

\begin{tabular}{|c|c|c|c|c|c|c|}
\hline Author, year & Country & Subjects & $\begin{array}{l}\text { Age/sex } \\
\text { matched }\end{array}$ & Results & $\begin{array}{l}\text { COPD as } \\
\text { DD risk }\end{array}$ & $\begin{array}{c}\text { Quality } \\
\text { score }\end{array}$ \\
\hline Kubota et al, 2016 & Japan & $\begin{array}{l}\text { Total: } 230 \\
\text { COPD: } 115\end{array}$ & $\mathrm{Y}$ & $\begin{array}{l}\text { High E/e' as an index of severe LVDD was significantly higher in the COPD } \\
\text { group than in the control group. E/e' significantly correlated to pulmonary } \\
\text { function tests. }\end{array}$ & $\mathrm{Y}$ & $2 / 1 / 2$ \\
\hline $\begin{array}{l}\text { Boussuges et al, } \\
2000\end{array}$ & France & $\begin{array}{l}\text { Total: } 54 \\
\text { COPD: } 34\end{array}$ & $\mathrm{Y}$ & $\begin{array}{l}\text { E/A ratio was significantly lower in patients with COPD (P: 0.02). The } \\
\text { transmitral flow pattern exhibited a dominant A wave with inverse E/A ratio } \\
\text { (E/A) in } 26 \text { patients with COPD ( } 76 \%) \text { and in only seven control subjects } \\
(35 \%) \text { (P: } 0.003 \text {, chi-square test). }\end{array}$ & $\mathrm{Y}$ & $3 / 1 / 2$ \\
\hline Farouk et al, 2017 & Egypt & $\begin{array}{l}\text { Total: } 53 \\
\text { COPD: } 35\end{array}$ & $\mathrm{Y}$ & $\begin{array}{l}\text { LV diastolic dysfunction was reported in } 20 \text { patients using the mitral inflow } \\
\text { indexes while in only } 12 \text { patients using the comprehensive approach }(\mathrm{P}=.021) \text {. }\end{array}$ & $\mathrm{Y}$ & $4 / 1 / 2$ \\
\hline Malerba et al, 2011 & Italy & $\begin{array}{l}\text { Total: } 95 ; \\
\text { COPD: } 55\end{array}$ & $\mathrm{Y}$ & $\begin{array}{l}\text { High E/e' as an index of severe LVDD was significantly higher in the COPD } \\
\text { group than in the control group. }\end{array}$ & $\mathrm{Y}$ & $4 / 1 / 2$ \\
\hline Huang et al, 2014 & China & $\begin{array}{l}\text { Total: } 148 \\
\text { COPD: } 75\end{array}$ & $\mathrm{Y}$ & $\begin{array}{l}\text { Compared with the control group, the } \mathrm{E} / \mathrm{e} \text { ratio was significantly higher in the } \\
\text { COPD group }(11.51 \pm 2.50 \text { vs } 10.42 \pm 3.25, \mathrm{P}=0.047) \text {. A high frequency of LVDD } \\
\text { was observed in patients with COPD }(65.6 \%) \text {, but there was no difference } \\
\text { among different stages of COPD. }\end{array}$ & $\mathrm{Y}$ & $3 / 1 / 2$ \\
\hline Eweda et al, 2015 & Egypt & $\begin{array}{l}\text { Total: } 60 \\
\text { COPD: } 40\end{array}$ & $\mathrm{Y}$ & $\begin{array}{l}\text { LVDD is related to the severity of COPD. Mitral A in the severe COPD } \\
\text { group }(\mathrm{P}<0.001) \text { was increased compared to the control. Lateral and septal } \\
\mathbf{E}^{\prime} \text { were also decreased }(\mathrm{p}<0.001) \text {. Mitral E/A in severe COPD }(\mathrm{P}<0.001) \text { was } \\
\text { decreased compared to the control. IVRT in the severe COPD group }(\mathrm{P}<0.001) \\
\text { was increased compared to the control. }\end{array}$ & $\mathrm{Y}$ & $3 / 1 / 2$ \\
\hline Faludi et al, 2016 & Hungary & $\begin{array}{l}\text { Total: } 99 ; \\
\text { COPD: } 65\end{array}$ & $\mathrm{Y}$ & $\begin{array}{l}\text { Lateral and septal } \mathrm{e}^{\prime} \text { were lower in the COPD group }(8.7 \pm 1.9 \text { and } 7.0 \pm 1.4) \\
\text { compared to the control group }(11.1 \pm 3.1 \text { and } 9.0 \pm 2.1) \text {. The mean LV E/e' was } \\
\text { higher in COPD patients }(8.8 \pm 2.1) \\
\text { vs controls }(6.4 \pm 1.4) \text {. E/A in the COPD group }(0.9 \pm 0.2) \text { was lower than in the } \\
\text { control group }(1.3 \pm 0.3) \text { LVDD was found in } 48(74 \%) \text { of COPD patients. LV } \\
\text { filling pressure was elevated in } 28(43 \%) \text { COPD patients. }\end{array}$ & $\mathrm{Y}$ & $4 / 1 / 2$ \\
\hline Sabit et al, 2010 & UK & $\begin{array}{l}\text { Total: } 50 \\
\text { COPD: } 36\end{array}$ & $\mathrm{Y}$ & $\begin{array}{l}\text { E/Ea of the COPD group }(10.7 \pm 2.6) \text { was increased compared to the control } \\
(7.9 \pm 1.6, \text { p }<0.01) \text {. IVRT of the COPD group }(125 \pm 15.2) \text { was increased } \\
\text { compared to the control }(98.2 \pm 21.1, \mathrm{p}<0.01) \text {. COPD patients had increased RV } \\
\text { myocardial relaxation time }(\mathrm{P}<0.001) \text {. Log } 10 \text {-IL- } 6 \text { was related to pulmonary } \\
\text { acceleration time and mitral annular E/A. }\end{array}$ & $\mathrm{Y}$ & $3 / 1 / 2$ \\
\hline Pela et al, 2016 & Italy & $\begin{array}{l}\text { Total: } 85 \\
\text { COPD: } 49\end{array}$ & $\mathrm{Y}$ & $\begin{array}{l}\text { The COPD patients had decreased LV size }(\mathrm{P}<0.05) \text { and increased relative } \\
\text { wall thickness }(\mathrm{RWT})(\mathrm{P}<0.001) \text { compared to controls, indicating concentric } \\
\text { remodeling of the left ventricle. RWT was significantly associated with FEV1/ } \\
\text { FVC and was the only cardiac parameter associated with COPD. RV } \mathbf{E}^{\prime} \text { tvi } \\
(\mathrm{p}=0.01) \text {, and } \mathbf{E}^{\prime} / \mathbf{A}^{\prime} \text { tvi }(\mathrm{p}<0.05) \text { were significantly decreased compared to the } \\
\text { control. }\end{array}$ & $\mathrm{Y}$ & $3 / 1 / 2$ \\
\hline Yilmaz et al, 2005 & Turkey & $\begin{array}{l}\text { Total: } 68 \\
\text { COPD: } 44\end{array}$ & $\mathrm{Y}$ & $\begin{array}{l}\text { Tricuspid E/A was decreased in COPD subjects }(\mathrm{p}<0.002) \text { and IVRT in } \\
\text { COPD patients w/ and w/o PH was increased compared to the control } \\
(\mathrm{p}<0.022,0.001, \text { respectively). By looking at LVMPI as a marker of both } \\
\text { diastolic and systolic function, both may be imparied in COPD, especially in } \\
\text { patients with PH. LVMPI was independently associated with PASP and FEV1. }\end{array}$ & $\mathrm{Y}$ & $4 / 1 / 3$ \\
\hline $\begin{array}{l}\text { El Wahsh et al, } \\
2013\end{array}$ & Egypt & $\begin{array}{l}\text { Total: } 48 ; \\
\text { COPD: } 36\end{array}$ & $\mathrm{Y}$ & $\begin{array}{l}\text { COPD patients had LVDD and LV global dysfunction. COPD patients had a } \\
\text { higher HR, less } \mathbf{E} \text { wave peak velocity }(\mathrm{p}<0.05) \text {, a smaller E/A ratio compared } \\
\text { to control subjects. There was a significant difference in E wave peak velocity } \\
\text { by flow and DTI, E/A by DTI, IVRT, and MPI between mild and very severe } \\
\text { COPD. There were statisticallhy significant changes in LVDD based on } \\
\text { severity of COPD. }\end{array}$ & $\mathrm{Y}$ & $4 / 1 / 3$ \\
\hline Funk et al, 2008 & US & $\begin{array}{l}\text { Total: } 44 ; \\
\text { COPD: } 22\end{array}$ & $\mathrm{Y}$ & $\begin{array}{l}\text { LV E max was decreased in COPD patients compared to controls }(\mathrm{p}<0.001) \text {. } \\
\text { A max was increased in COPD patients }(\mathrm{p}<0.0001) \text {. E/A was decreased in } \\
\text { COPD patients }(\mathrm{p}<0.0001) \text {. Observed correlation between E/A and A\% } \\
\text { with FEV1 and } \mathrm{PaO} 2 . \text { Observed correlation between mPAP and E/A ratio. } \\
\text { Observed that COPD patients with and without pulmonary hypertension had } \\
\text { impaired LV diastolic filling. }\end{array}$ & $\mathrm{Y}$ & $3 / 1 / 3$ \\
\hline Suchon et al, 2007 & Poland & $\begin{array}{l}\text { Total: } 60 ; \\
\text { COPD: } 35\end{array}$ & $\mathrm{Y}$ & $\begin{array}{l}\text { E/A was significantly lower in COPD patients }(\mathrm{p}<0.001) \text {. E/A and RVSP were } \\
\text { inversely correlated }(-0.61 ; \mathrm{p}<0.001) \text {. IVRT was significantly longer compared } \\
\text { to the control }(\mathrm{p}<0.001) \text {. }\end{array}$ & $\mathrm{Y}$ & $3 / 1 / 2$ \\
\hline Acharya et al, 2013 & India & Total: 100 & $\mathrm{Y}$ & $\begin{array}{l}\text { There were no statistically significant differences between Mitral E/A among } \\
\text { the studied groups ( } \mathrm{p}=0.183 \text { ). There was a statistically significant difference } \\
\text { between Septal } E / \mathbf{E}^{\prime} \text { and Lateral E/E' between cases and controls ( } \mathrm{p}=0.003 \text { and } \\
\mathrm{p}<0.001 \text { respectively). Prolonged IVRT in COPD group compared to controls. }\end{array}$ & $\mathrm{Y}$ & $2 / 1 / 2$ \\
\hline Abo El-Magd, 2017 & Egypt & $\begin{array}{l}\text { Total: } 80 \\
\text { COPD: } 60\end{array}$ & $\mathrm{Y}$ & $\begin{array}{l}\text { Regarding IVRT, DT of the early transmitral flow, E and A waves; there was } \\
\text { significant statstical significance between COPD stage III/IV cmpared to stage } \\
\text { stage } 1 / \mathrm{II} \text { and compared to the control group. }\end{array}$ & $\mathrm{Y}$ & $3 / 1 / 2$ \\
\hline
\end{tabular}

Table 1: Major features of studies included in the analysis.

LVDD - left ventricular diastolic dysfunction; COPD - chronic obstructive pulmonary disease; LV - left ventricle; IVRT - isovolumic relaxation time; RV - right ventricle; RWT - relative wall thickness; LVMPI- left ventricular myocardial performance index; PASP - pulmonary artery systolic pressure; FEV1 - forced epiratory volume; DTI - diffusion tensor imaging; mPAP - mean pulmonary arterial pressure; RVSP -right ventricular systolic pressure, DT - deceleration time. 
Citation: Zhyvotovska A, Yusupov D, Kamran H, Al-Bermani T, Abdul R, et al. (2019) Diastolic Dysfunction in Patients with Chronic Obstructive Pulmonary Disease: A Meta-Analysis of Case Controlled Studies. Int J Clin Res Trials 4: 137. doi: https://doi.org/10.15344/2456-8007/2019/137

\section{E/A Ratio}

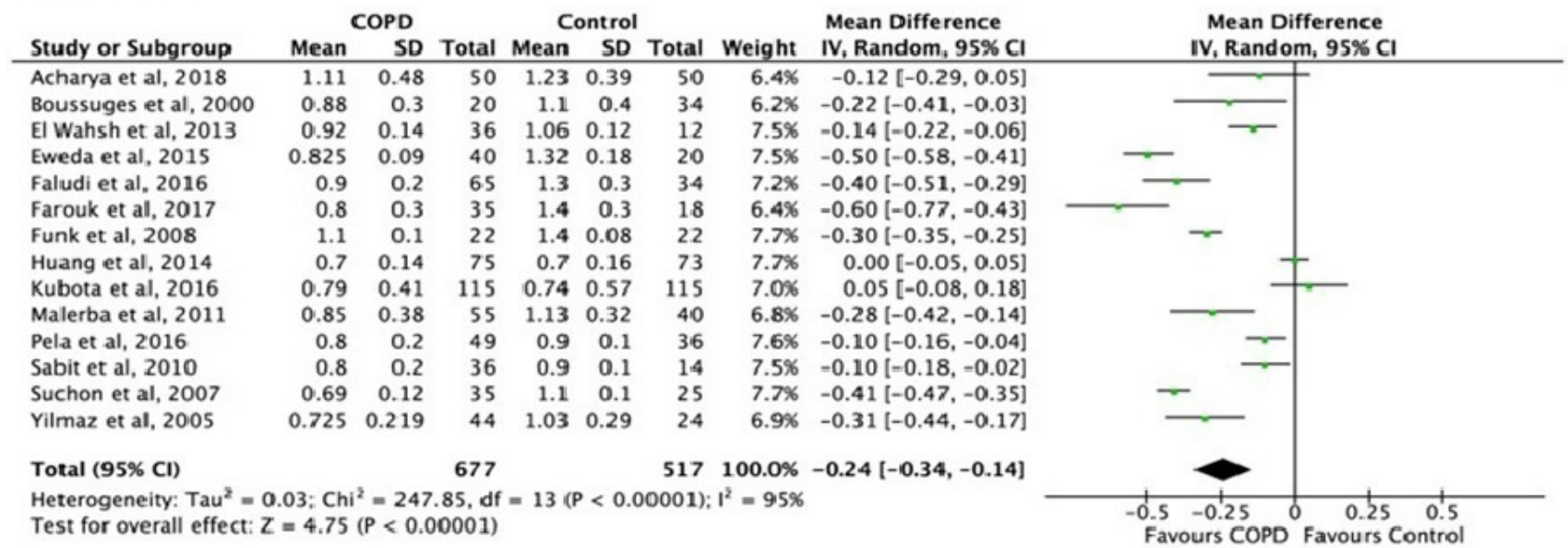

\section{IVRT (Isovolumetric relaxation time) msec}

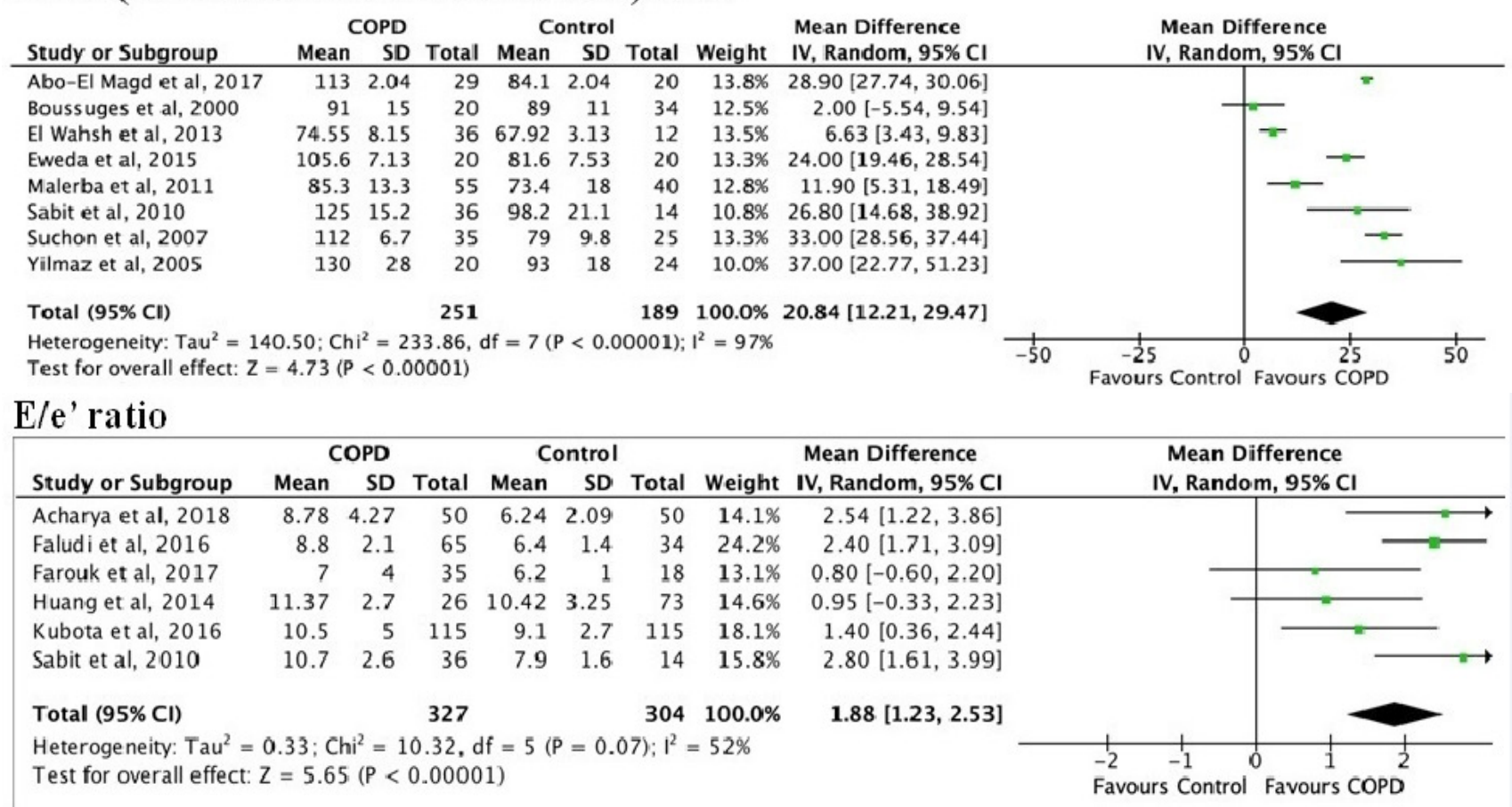

\section{A wave peak velocity (Apv)(meters/second)}

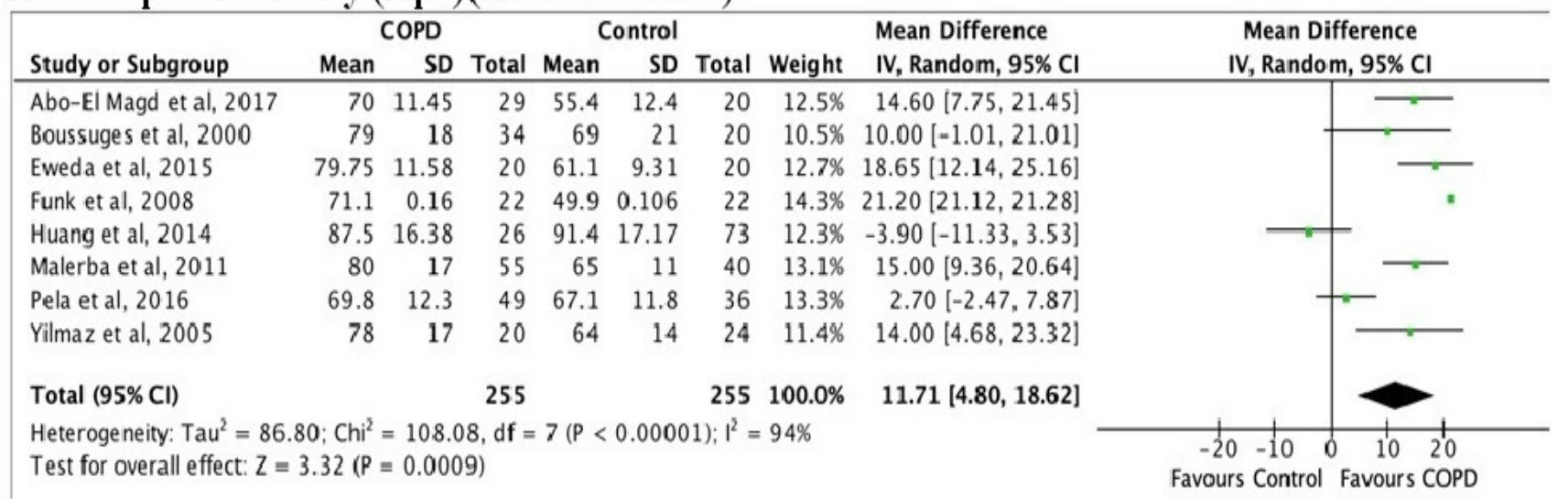

Continue... 
Citation: Zhyvotovska A, Yusupov D, Kamran H, Al-Bermani T, Abdul R, et al. (2019) Diastolic Dysfunction in Patients with Chronic Obstructive Pulmonary Disease: A Meta-Analysis of Case Controlled Studies. Int J Clin Res Trials 4: 137. doi: https://doi.org/10.15344/2456-8007/2019/137

E wave peak velocity (Epv)(meters/second)

\begin{tabular}{|c|c|c|c|c|c|c|c|c|c|c|}
\hline \multirow[b]{2}{*}{ Study or Subgroup } & \multicolumn{3}{|c|}{ COPD } & \multicolumn{3}{|c|}{ Control } & \multicolumn{2}{|r|}{ Mean Difference } & \multirow{2}{*}{\multicolumn{2}{|c|}{$\begin{array}{l}\text { Mean Difference } \\
\text { IV, Random, } 95 \% \mathrm{Cl}\end{array}$}} \\
\hline & Mean & SD & Total & Mean & SD & Total & Weight & IV, Random, 95\% CI & & \\
\hline Abo-El Magd et al, 2017 & 67.41 & 7.58 & 29 & 78.3 & 21.01 & 20 & $8.1 \%$ & $-10.89[-20.50,-1.28]$ & & \\
\hline Boussuges et al, 2000 & 67 & 22 & 34 & 70 & 19 & 20 & $7.3 \%$ & $-3.00[-14.14,8.14]$ & & \\
\hline El Wahsh et al, 2013 & 54 & 12 & 36 & 64 & 22 & 12 & $6.4 \%$ & $-10.00[-23.05,3.05]$ & & \\
\hline Eweda et al, 2015 & 64.85 & 14.26 & 20 & 79.9 & 9.8 & 20 & $9.1 \%$ & $-15.05[-22.63,-7.47]$ & & \\
\hline Farouk et al, 2017 & 50 & 10 & 35 & 80 & 20 & 18 & $8.0 \%$ & $-30.00[-39.82,-20.18]$ & & \\
\hline Funk et al, 2008 & 55.2 & 0.12 & 22 & 67.6 & 0.14 & 22 & $11.7 \%$ & $-12.40[-12.48,-12.32]$ & 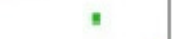 & \\
\hline Huang et al, 2014 & 61 & 11.59 & 26 & 63.21 & 14.1 & 73 & $10.2 \%$ & $-2.21[-7.72,3.30]$ & & \\
\hline Kubota et al, 2016 & 58.7 & 18.5 & 115 & 53.3 & 16.3 & 115 & $10.6 \%$ & $5.40[0.89,9.91]$ & & $\longrightarrow$ \\
\hline Malerba et al, 2011 & 62 & 14 & 55 & 71 & 11 & 40 & $10.4 \%$ & $-9.00[-14.03,-3.97]$ & & \\
\hline Pela et al, 2016 & 53.6 & 13.6 & 49 & 58.3 & 13.2 & 36 & $10.1 \%$ & $-4.70[-10.45,1.05]$ & & \\
\hline Yilmaz et al, 2005 & 57 & 18 & 20 & 66 & 13 & 24 & $8.1 \%$ & $-9.00[-18.45,0.45]$ & & \\
\hline Total $(95 \%$ CI) & & & 441 & & & 400 & $100.0 \%$ & $-8.74[-13.63,-3.85]$ & & \\
\hline \multicolumn{9}{|c|}{$\begin{array}{l}\text { Heterogeneity: } \mathrm{Tau}^{2}=53.12 ; \mathrm{Chi}^{2}=97.96, \mathrm{df}=10(\mathrm{P}<0.00001) ; \mathrm{I}^{2}=90 \% \\
\text { Test for overall effect: } \mathrm{Z}=3.51(\bar{P}=0.0005)\end{array}$} & $\begin{array}{l}-20-10 \\
\text { Favours COPD }\end{array}$ & $\begin{array}{ll}10 & 20 \\
\text { Favours COntrol }\end{array}$ \\
\hline
\end{tabular}

\section{e' lateral $(\mathrm{cm} / \mathrm{sec})$}

\begin{tabular}{lrrrrrrrrr} 
& \multicolumn{2}{c}{ COPD } & \multicolumn{4}{c}{ Control } & & Mean Difference \\
Study or Subgroup & Mean & SD & Total & Mean & SD & Total & Weight & IV, Random, 95\% CI \\
\hline Eweda et al, 2015 & 6.37 & 0.56 & 20 & 12.24 & 1.7 & 20 & $25.6 \%$ & $-5.87[-6.65,-5.09]$ \\
Faludi et al, 2016 & 8.7 & 1.9 & 65 & 11.1 & 3.1 & 34 & $25.1 \%$ & $-2.40[-3.54,-1.26]$ \\
Farouk et al, 2017 & 10 & 4 & 35 & 13 & 3 & 18 & $23.6 \%$ & $-3.00[-4.92,-1.08]$ \\
Kubota et al, 2016 & 7.7 & 2.3 & 115 & 7.8 & 3 & 115 & $25.7 \%$ & $-0.10[-0.79,0.59]$ \\
Total (95\% Cl) & & & & 235 & & & 187 & $100.0 \%$ & $-2.84[-5.91,0.24]$
\end{tabular}

Heterogeneity: $\mathrm{Tau}^{2}=9.46 ; \mathrm{Chi}^{2}=117.34, \mathrm{df}=3(\mathrm{P}<0.00001) ; \mathrm{I}^{2}=97 \%$

Test for overall effect: $Z=1.81(P=0.07)$

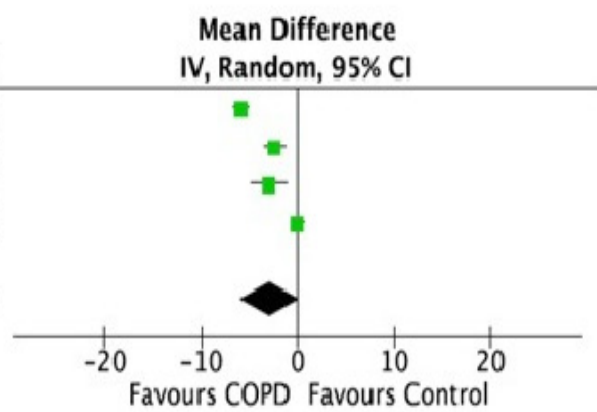

e'septal $(\mathrm{cm} / \mathrm{sec})$

COPD

Control

Mean Difference

Mean Difference

Study or Subgroup Mean SD Total Mean SD Total Weight IV, Random, $95 \% \mathrm{CI}$

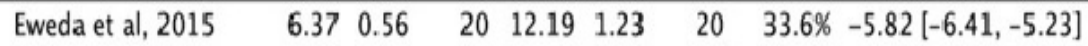

$\begin{array}{llllllllll}\text { Faludi et al, } 2016 & 7 & 1.4 & 65 & 9 & 2.1 & 34 & 33.4 \% & -2.00 & {[-2.78,-1.22}\end{array}$

$\begin{array}{lllllllll}\text { Kubota et al, } 2016 \quad 5.9 & 1.7 & 115 & 6.1 & 5.3 & 115 & 33.0 \% & -0.20 & {[-1.22,0.82]}\end{array}$

Total $(95 \% \mathrm{Cl}) \quad 200 \quad 169100.0 \%-2.69[-6.07,0.69]$

Heterogeneity: $\mathrm{Tau}^{2}=8.75 ; \mathrm{Chi}^{2}=112.76, \mathrm{df}=2(\mathrm{P}<0.00001) ; \mathrm{I}^{2}=98 \%$

Test for overall effect: $Z=1.56(P=0.12)$

IV, Random, 95\% Cl

\section{$\underline{\text { Right ventricular end diastolic diameter (RVEDD) (mm) }}$}

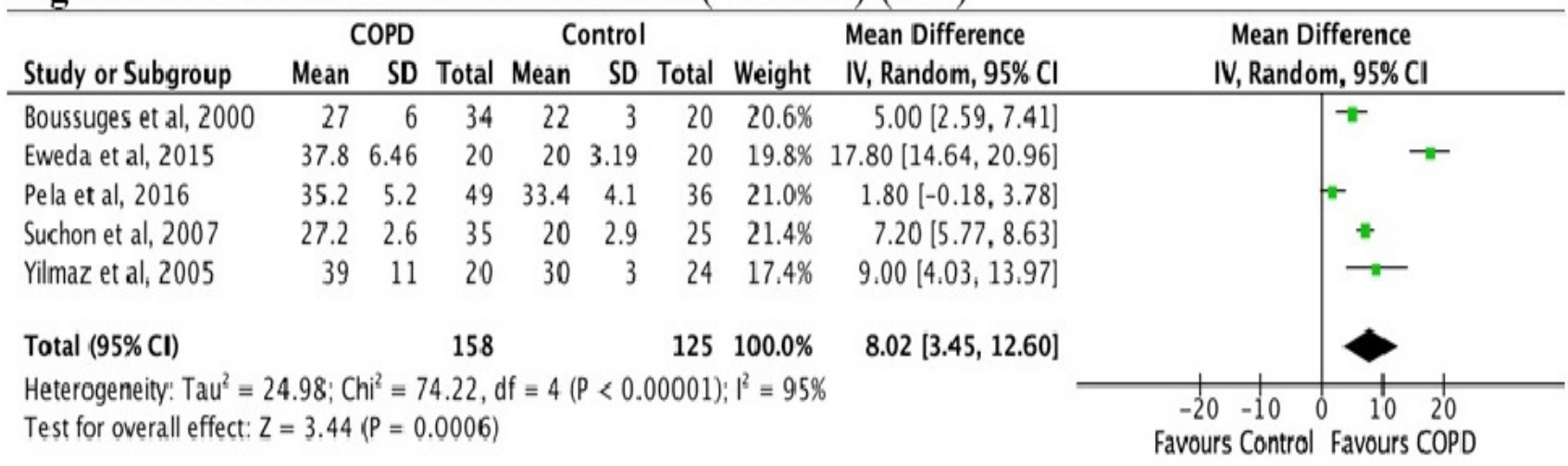

Continue.. 
Citation: Zhyvotovska A, Yusupov D, Kamran H, Al-Bermani T, Abdul R, et al. (2019) Diastolic Dysfunction in Patients with Chronic Obstructive Pulmonary Disease: A Meta-Analysis of Case Controlled Studies. Int J Clin Res Trials 4: 137. doi: https://doi.org/10.15344/2456-8007/2019/137

Page 6 of 11

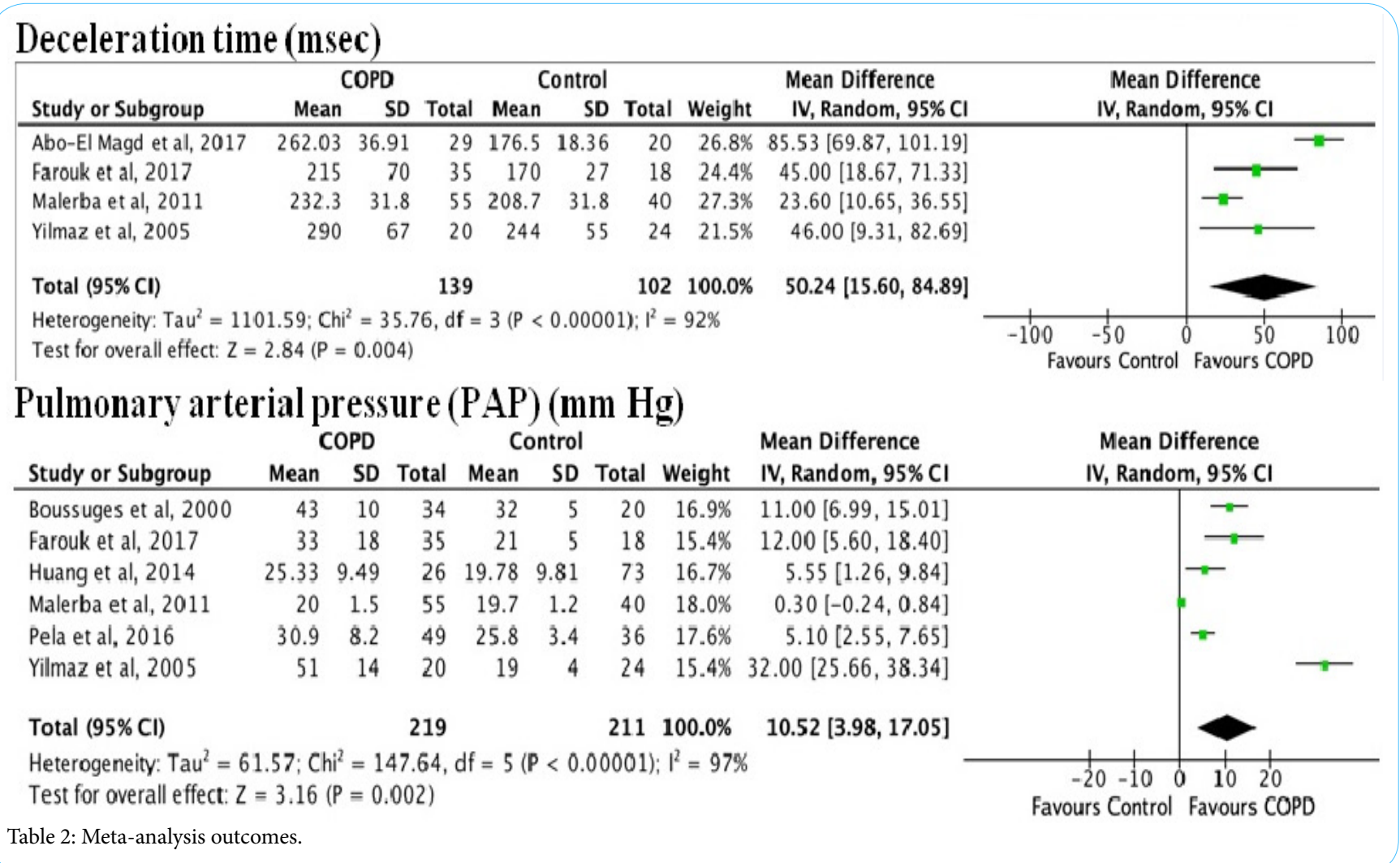

All of the studies were age and sex-matched. Many of the following were excluded from the studies: 1. Subjects with asthma, hypoxia, pneumonia or patients in acute exacerbation period; 2 . Subjects with $\mathrm{DM}, \mathrm{CAD}$, hypertension, valvular pathology, left ventricular systolic dysfunction (LVSD) or arrhythmia; 3. Subjects with diffuse lung parenchymal disease, lung malignancies; 4 . Subjects with renal failure. Three studies included patients with confounding comorbidities $[22,25,26]$. The key points of the individual studies are represented in the Table 1 . The demographic information for each study can be found in Table 3.

Our meta-analysis found the prevalence of LVDD echocardiographic parameters were higher among COPD subjects versus the control group. We analyzed the most commonly represented LVDD parameters. Patients with COPD had prolonged IVRT (mean difference 20.84 [95\% CI 12.21, 29.47]; $\mathrm{P}<0.00001$ ), lower E/A ratio (mean difference - 0.24 [95\% CI -0.34, 00.14]; P < 0.00001), higher transmitral A wave peak velocity (Apv) (mean difference 11.71 [95\% CI $4.80,18.62] ; \mathrm{P}<0.00001$ ), higher $\mathrm{E} / \mathrm{e}^{\prime}$ ratio (mean difference 1.88 [95\% CI 1.23, 2.53]; $\mathrm{P}<0.00001)$, lower mitral $\mathrm{E}$ wave peak velocity (Epv) (mean difference -8.74 [95\% CI -13.63, -3.85]; $\mathrm{P}<$ 0.0005 ), prolonged deceleration time (mean difference 50.24 [95\% CI 15.60, 84,89]; $\mathrm{P}<0.004)$, a higher right ventricular end diastolic diameter (RVEDD) (mean difference 8.02 [95\% CI 3.45, 12.60]; $\mathrm{P}<$ 0.0006 ) compared to controls. Differences in septal e' velocity (mean difference -2.69 [95\% CI $-6.07,0.69] ; \mathrm{P}=0.12$ ) and in lateral e' velocity (mean difference -2.84 [95\% CI 5.91, 0.24]; $\mathrm{P}=0.07$ ) trended towards significance but did not meet our cutoff for statistical significance $(\mathrm{p}<$ 0.05).COPD patients had a higher pulmonary arterial pressure (PAP) (mean difference 10.52 [95\% CI 3.98, 17.05]; $\mathrm{P}<0.002$ ). The details of the individual studies are presented in Table 2.
Publication bias was assessed using a funnel-plot. There was no evidence of publication bias in our study. The plot showed a funnel-like shape (Figure 2), with estimated spanning down from the larger trials in both directions with increasing variability. The Figure 2 showed symmetric distributions in both directions and around midline. Given the large heterogeneity, each echocardiographic variable was analyzed separately using the random effects model to calculate the mean difference and 95\% CI.

\section{Discussion}

To our knowledge, this is the first systematic meta-analysis on all case-control studies since the year 2000 looking at diastolic dysfunction in the COPD population. Our review, involving 1,403 patients, reveals that patients with COPD are more likely to have features of LVDD by transmitral inflow patterns and tissue doppler. Compared to the control group, the COPD group had significantly higher E/e', lower E/A ratio, higher A wave peak velocity, lower E wave peak velocities, and greater IVRT and DT. When taken together, this information suggests that patients with COPD have a greater prevalence of LVDD indices and thus may be prone to develop HFpEF. At least one echocardiographic parameter in all of the 15 studies was statistically different in the COPD group compared to the control group.

Our findings are of clinical significance as concomitant cardiovascular disease (CVD) plays a role in determining the clinical picture in patients with COPD. There is, for example, a $28 \%$ increase in cardiovascular mortality for every $10 \%$ decrease in FEV1 in COPD [33]. Even when confounders such as exposure to smoke are accounted for, COPD patients have been linked to a 2-3 fold increase in cardiovascular (CV) events [33]. 
Citation: Zhyvotovska A, Yusupov D, Kamran H, Al-Bermani T, Abdul R, et al. (2019) Diastolic Dysfunction in Patients with Chronic Obstructive Pulmonary Disease: A Meta-Analysis of Case Controlled Studies. Int J Clin Res Trials 4: 137. doi: https://doi.org/10.15344/2456-8007/2019/137

Page 7 of 11

\begin{tabular}{|c|c|c|c|c|c|c|c|c|}
\hline & $\begin{array}{l}\text { Eweda et. al, } \\
2015\end{array}$ & \begin{tabular}{|l} 
Sabit et. al, \\
2010
\end{tabular} & $\begin{array}{l}\text { Yilmaz et al, } \\
2005\end{array}$ & $\begin{array}{l}\text { Wahsh et al, } \\
2013\end{array}$ & $\begin{array}{l}\text { Funk et al, } \\
2008\end{array}$ & $\begin{array}{l}\text { Suchon et al, } \\
2007\end{array}$ & $\begin{array}{l}\text { Abo-El Magd } \\
\text { et al, } 2017\end{array}$ & $\begin{array}{l}\text { Acharya et } \\
\text { al, } 2018\end{array}$ \\
\hline Control total & 20 & 14 & 24 & 12 & 22 & 25 & 20 & 50 \\
\hline COPD total & 40 & 36 & 44 & 36 & 22 & 35 & 60 & 50 \\
\hline Control average age & $51.40+/-3.89$ & $67+/-8.8$ & $63+/-12$ & $51.9+/-9.7$ & $54.8+/-1.3$ & $61.6+/-10.1$ & $57.42+/-6.44$ & $\star$ \\
\hline COPD average age & $51+/-5$ & $66.5+/-8.9$ & $64.4+/-8$ & $55.1+/-7.3$ & $59.1+/-1.7$ & $62.1+/-7.7$ & $57.73+/-5.93$ & * \\
\hline Control male n (\%) & 10 & 9 & $13(65)$ & $6(50)$ & $\mathrm{n} / \mathrm{a}$ & $18(72)$ & 12 & $*$ \\
\hline COPD male n (\%) & 19 & 19 & 31 & $30(83.3)$ & $\mathrm{n} / \mathrm{a}$ & $25(71)$ & 42 & * \\
\hline Control average BMI & $\mathrm{n} / \mathrm{a}$ & $26.9+/-3.2$ & $\mathrm{n} / \mathrm{a}$ & $\mathrm{n} / \mathrm{a}$ & $\mathrm{n} / \mathrm{a}$ & $\mathrm{n} / \mathrm{a}$ & $\mathrm{n} / \mathrm{a}$ & * \\
\hline COPD average BMI & $\mathrm{n} / \mathrm{a}$ & $27.3+/-4.9$ & $\mathrm{n} / \mathrm{a}$ & $\mathrm{n} / \mathrm{a}$ & $\mathrm{n} / \mathrm{a}$ & $\mathrm{n} / \mathrm{a}$ & $\mathrm{n} / \mathrm{a}$ & * \\
\hline Control HTN n (\%) & 0 & $\mathrm{n} / \mathrm{a}$ & 0 & $\mathrm{n} / \mathrm{a}$ & 0 & 0 & $\mathrm{n} / \mathrm{a}$ & 0 \\
\hline COPD HTN n (\%) & 0 & $\mathrm{n} / \mathrm{a}$ & 0 & $\mathrm{n} / \mathrm{a}$ & 0 & 0 & $\mathrm{n} / \mathrm{a}$ & 0 \\
\hline Control DM n (\%) & 0 & $\mathrm{n} / \mathrm{a}$ & $\mathrm{n} / \mathrm{a}$ & $\mathrm{n} / \mathrm{a}$ & 0 & $\mathrm{n} / \mathrm{a}$ & 0 & 0 \\
\hline COPD DM n (\%) & 3 & $\mathrm{n} / \mathrm{a}$ & $\mathrm{n} / \mathrm{a}$ & $\mathrm{n} / \mathrm{a}$ & 0 & $\mathrm{n} / \mathrm{a}$ & 0 & 0 \\
\hline Control CVD n (\%) & 0 & $\mathrm{n} / \mathrm{a}$ & 0 & 0 & 0 & 0 & 0 & 0 \\
\hline COPD CVD n (\%) & 0 & $\mathrm{n} / \mathrm{a}$ & 0 & 0 & 0 & 0 & 0 & 0 \\
\hline Control CKD n (\%) & 0 & $\mathrm{n} / \mathrm{a}$ & $\mathrm{n} / \mathrm{a}$ & $\mathrm{n} / \mathrm{a}$ & $\mathrm{n} / \mathrm{a}$ & $\mathrm{n} / \mathrm{a}$ & $\mathrm{n} / \mathrm{a}$ & $\mathrm{n} / \mathrm{a}$ \\
\hline COPD CKD n (\%) & 0 & $\mathrm{n} / \mathrm{a}$ & $\mathrm{n} / \mathrm{a}$ & $\mathrm{n} / \mathrm{a}$ & $\mathrm{n} / \mathrm{a}$ & $\mathrm{n} / \mathrm{a}$ & $\mathrm{n} / \mathrm{a}$ & $\mathrm{n} / \mathrm{a}$ \\
\hline \multicolumn{9}{|c|}{${ }^{*}$ Author did not provide continuous demographic information } \\
\hline & $\begin{array}{l}\text { Kubota et al, } \\
2016\end{array}$ & $\begin{array}{l}\text { Boussuges et } \\
\text { al, } 2000\end{array}$ & \begin{tabular}{|l|} 
Farouk et al, \\
2017
\end{tabular} & $\begin{array}{l}\text { Malerba et al, } \\
2011\end{array}$ & $\begin{array}{l}\text { Huang et al, } \\
2014\end{array}$ & $\begin{array}{l}\text { Faludi et al, } \\
2016\end{array}$ & Pela et al, 2016 & \\
\hline Control total & 115 & 20 & 18 & 40 & 73 & 34 & 36 & \\
\hline COPD total & 115 & 34 & 35 & 55 & 75 & 65 & 49 & \\
\hline Control average age & 74.4 & $60+/-10$ & $56+/-8$ & $55.9+/-11.4$ & $78.67 \pm 7.14$ & $58+/-8.3$ & 62.7 & \\
\hline COPD average age & 74.4 & $58+/-11$ & $58.9+/-9$ & $58.8+/-11.5$ & $82.76 \pm 5.71$ & $60.8+/-9$ & 66.3 & \\
\hline Control male n (\%) & $72.3(65)$ & $12(60)$ & 100 & $21(52)$ & $51(69.9)$ & $18(53)$ & $24(69)$ & \\
\hline COPD male n (\%) & $72.3(65)$ & $21(61.7)$ & 100 & $23(52)$ & $60(80.0)$ & $39(60)$ & $37(76)$ & \\
\hline Control average BMI & 21.5 & wt. $65+/-17$ & $26+/-5$ & $27.7+/-4.4$ & $23.92 \pm 3.10$ & $\mathrm{n} / \mathrm{a}$ & \begin{tabular}{|l|l|}
25.8 \\
\end{tabular} & \\
\hline COPD average BMI & 22.5 & wt. $76+/-15$ & 25.6 & $28.4+/-4.2$ & $23.56 \pm 3.10$ & $\mathrm{n} / \mathrm{a}$ & 25.3 & \\
\hline Control HTN n (\%) & $64(55.4)$ & 0 & 0 & 0 & $41(56.2)$ & $\mathrm{n} / \mathrm{a}$ & & \\
\hline COPD HTN n (\%) & $53(45.5)$ & 0 & 0 & 0 & $52(69.3)$ & $51(78)$ & 0 & \\
\hline Control DM n (\%) & 20.5 & 0 & 0 & 0 & $27(36.9)$ & $\mathrm{n} / \mathrm{a}$ & 0 & \\
\hline COPD DM n (\%) & 25 & 0 & 0 & 0 & $17(45.4)$ & $18(28)$ & 3 & \\
\hline Control CVD n (\%) & 0 & 0 & 0 & 0 & $32(43.8)$ & 0 & 0 & \\
\hline COPD CVD n (\%) & 0 & 0 & 0 & 0 & $29(38.7)$ & $9(12)$ & 0 & \\
\hline Control CKD n (\%) & 38.4 & 0 & 0 & $\mathrm{n} / \mathrm{a}$ & $12(16.4)$ & $\mathrm{n} / \mathrm{a}$ & $\mathrm{n} / \mathrm{a}$ & \\
\hline COPD CKD n (\%) & 41.4 & 0 & 0 & $\mathrm{n} / \mathrm{a}$ & $14(18.7)$ & $\mathrm{n} / \mathrm{a}$ & $\mathrm{n} / \mathrm{a}$ & \\
\hline
\end{tabular}

Table 3: Characteristics of study subjects.

\section{Funnel Plot of Standard Error by Hedges's g}

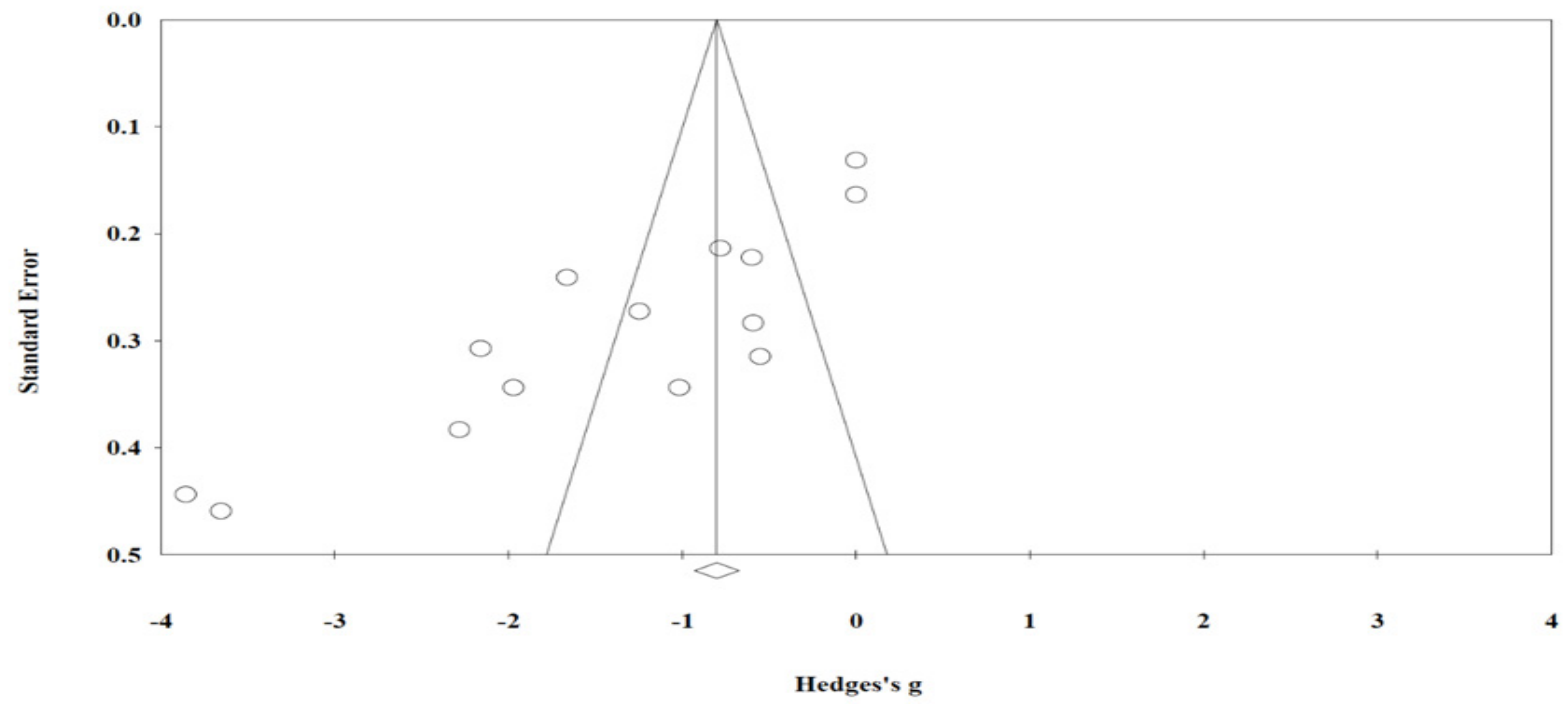

Figure 2: Funnel plot to assess for evidence of publication bias in our study. 
Citation: Zhyvotovska A, Yusupov D, Kamran H, Al-Bermani T, Abdul R, et al. (2019) Diastolic Dysfunction in Patients with Chronic Obstructive Pulmonary Disease: A Meta-Analysis of Case Controlled Studies. Int J Clin Res Trials 4: 137. doi: https://doi.org/10.15344/2456-8007/2019/137

Page 8 of 11

Furthermore, Finkelstein et al., found that the diagnosis of COPD increases the risk of having CVD independently of smoking history, age, gender, lifestyle, and co-morbid risk factors [34]. Interestingly, they also found that COPD reveals the strongest association, greater than that of other cardiovascular diseases, with heart failure [34]. Although the role of coexistent LVDD in CV morbidity and mortality is unknown in the COPD population, in patients with HFpEF rates of hospitalization and death approach those of HFrEF [10,35] .Thus, in the COPD population, HFpEF which may result from LVDD may have an additive detrimental effect [35-36].

Doppler indices to diagnose LVDD are imperfect, and no one parameter has sufficient sensitivity and specificity to diagnose LVDD. However, the more parameters that are abnormal, the more suggestive the diagnosis. The American Society of Echocardiography recommends a comprehensive approach [17]. Evidence of high left ventricular filling pressures are necessary in diagnosing HFpEF [17,37]. A frequently used Doppler parameter is the E/e' ratio. A higher E/e' correlates with an elevated left ventricular diastolic pressure, and is the recommended starting point in the diastolic evaluation for patients with normal ejection fraction [17,37]. When the ratio is between 8 and 15, it is important to combine this value with other diastolic parameters. The benefits of the E/e' ratio is that it is less age dependent, is not influenced by preload and heart rate, is an early marker of LVDD, and provides prognostic information and is very rarely $>14$ in normal persons $[17,37]$. Our study found a mean difference in $\mathrm{E} / \mathrm{e}$ ' of 1.88 , greater in the COPD group of patients.

Normal cardiac time intervals indicate normal cardiac function. Thus, time intervals play an important role in assessing for LVDD. As diastolic function declines, early diastolic relaxation proceeds moreslowly, leading to a prolonged IVRT $[17,37,38]$. The benefit of IVRT is that it provides assessment of the earliest phase of diastole, measuring the interval between aortic valve closure and mitral valve opening [37]. However, it is influenced by tachycardia and arterial pressure and may shorten with increased left a trial pressure, as expected in later stages of LVDD. In our study, there was a mean difference of $20 \mathrm{msec}$ favoring the COPD group, indicating that early diastolic filling is more likely to be impaired in patients with COPD.

Filling patterns such as the E/A ratio and deceleration time (dt) are feasible, reproducible and provide prognostic information [17]. With impaired relaxation, one would expect an increased $\mathrm{dt}(>220 \mathrm{~ms})$ and an inverse E/A ratio $(<1)$. This pattern has a high specificity for abnormal left ventricular (LV) relaxation and can be seen with normal or increased filling pressures. As diastolic dysfunction worsens in severity, the E/A ratio may normalize, known as pseudonormalization, or even become greater than 2.Similarly, as LVDD worsens LV pressures will reach a point halting early E flow abruptly, causing a decrease in deceleration time. However, impaired relaxation is the earliest step and is expected in all patients with diastolic dysfunction [6]. The E/A ratio in the COPD group in our study was significantly reduced and the deceleration time prolonged, indicating that filling patterns may be consistent with impaired relaxation.

The mechanism(s) of why echo parameters of LVDD are more common in the COPD population are speculative. Possible influences include tachycardia, systemic inflammation, pulmonary hypertension, chronic hypoxemia, chronic hypercapnia, hyperinflation, and right ventricular - left ventricular interaction.
Our study demonstrates that patients with COPD have a tendency towards higher left ventricular filling pressures, as evidenced by the increased E/e' compared to controls, and worse early diastolic filling as evidenced by lower E/A ratio, lower Epv, higher Apv, and increased IVRT and DT. COPD may lead to pulmonary hypertension, right ventricular hypertrophy and dilation, which may compromise left ventricular filling.

Heart rate (HR) was increased in nearly all of the reviewed COPD groups. Tachycardia particularly effects parameters obtained by doppler echocardiography. Tachycardia can affect E/A ratio, IVRT and DT, however, all but a few studies adjusted their data for HR, or controlled for HR, and yet differences indicating DD persisted. The causes of tachycardia in COPD are manifold; they include side effects of therapy, chronic hypoxia, hypercapnia, and autonomic dysregulation [31,39]. Boussuges et al., found ultrasonographic evidence of decreased LV filling in their COPD group, indicated by a significant decline in the total transmitral VTI [20]. Hyperinflation as a cause of decreased preload in the COPD population has been found in other studies [40-42]. Thus, decreased preload in patients with COPD may also contribute to tachycardia.

Lung hyperinflation in LVDD in the COPD population may be important. Lung hyperinflation and distension may lead to increased stiffness of the parietal pleura, which induces pressure onto the walls of the cardiac fossa. This in turn adds load onto the LV by necessitating conformational changes of the heart during the cardiac cycle [24,31]. This stiffness, imposed upon the heart by the parietal pleura and the cardiac fossa, may be one of the explanations of LVDD in COPD patients irrespective of pulmonary artery pressures.

In addition to lung hyperinflation, the presence of airway obstruction may provoke DD [27]. Hyperinflation and airway obstruction, by increasing positive end-expiratory pressure, may lead to decreased pulmonary vascular compliance, increased right ventricular load, reduced right ventricular stroke volume and, thus, reduced left ventricular filling [21]. Kubota et al. found that E/e' significantly correlated with patients' residual volume/total lung capacity ratio [26]. This strengthens the theory that hyperinflation is likely a contributing factor to elevated left ventricular pressures and diastolic dysfunction, and the severity of COPD may relate to severity of LVDD.

Pulmonary hypertension is a common manifestation of long standing or severe COPD, and has been linked to significant effects on morbidity and survival $[43,44]$.Yilmaz et al. found that LV abnormalities were related to severity of COPD, increased PAP and deterioration of RV function [32]. Suchon et al., also found a strong correlation between the severity of diastolic dysfunction and the severity of the PASP [30]. Sabit et al. found that $\mathrm{FEV}_{1}$ was related to indices of pulmonary hypertension such as pulmonary acceleration time, right ventricular free wall thickness, and myocardial relaxation time. Not surprisingly, pulmonary artery pressure was significantly elevated in the COPD population in our study. Some of same mechanisms which lead to the pulmonary vascular changes in COPD may also contribute to LVDD such as endothelial damage, systemic inflammation and chronic hypoxia causing increased endothelin synthesis and release, and decreased NO and prostacyclin synthesis and release among other mechanisms, and are linked to arterial stiffness [45]. 
Citation: Zhyvotovska A, Yusupov D, Kamran H, Al-Bermani T, Abdul R, et al. (2019) Diastolic Dysfunction in Patients with Chronic Obstructive Pulmonary Disease: A Meta-Analysis of Case Controlled Studies. Int J Clin Res Trials 4: 137. doi: https://doi.org/10.15344/2456-8007/2019/137

Page 9 of 11

Additionally, chronic hypoxemia may cause calcium transport disturbances leading to myocyte hypoxia and abnormalities of myocardial relaxation $[24,46]$. The interplay between hypoxemia and interventricular dependence was pointed out in the study by Sabit et al., which found that arterial oxygen tension was related to right ventricular (RV) free wall thickness and myocardial relaxation time [29].

Systemic inflammation is a known contributor to the development of HFpEF. Pro-inflammatory states, such as COPD, lead to an increase in proinflammatory markers such as IL- 6 and TNF- $\alpha$, among others. In the coronary microvasculature this leads to an increase in E-selectin, VCAM and endothelial Reactive oxygen species (ROS) expression and a decrease in nitric oxide (NO) availability [47]. These processes, through further signaling (NO-cGMP-PKG) lead to an increase of collagen deposition via myofibroblasts. Collectively these processes may lead to compromised coronary microvasculature and stiffer myocardial tissue [47]. IL-6 levels were significantly greater in the study performed by Sabit et al. [29]. Additionally, the levels of IL-6 were related to pulmonary acceleration time, LV strain and mitral annular e/a [29].

Arterial stiffness plays an important role in LVDD. In the study by Sabit et al., they found that the COPD group had a $22 \%$ greater pulse wave velocity (PWV) [29]. They also found the PWV was related to LVDD parameters such as E/e'; mitral E/A; mitral annular e/a and IVRT. However, they did not find any relationship between PWV and parameters of systolic function. Thus arterial stiffness may manifest in COPD and contribute to LVDD. PWV is a simple and reproducible method of determining arterial stiffness [48]. Arterial stiffness leads to a left ventricular afterload increases because of the earlier return of the reflected arterial wave causing increased left ventricular pressures, increased myocardial oxygen demand, and concomitant reduction in diastolic blood pressures, itself a cause of subendocardial ischemia with resulting myocardial relaxation abnormalities [25,48]. The magnitude of wave reflection is associated with DD [17]. Endothelial dysfunction is a known consequence of COPD seen throughout the disease course, from early to end-stage disease, and is directly linked to irreversible eNOS inhibition by cigarette smoke [44].

Patients with both pulmonary hypertension and LVDD may be more susceptible to dyspnea. The right ventricle is thinner and weaker than the left, meaning it has difficulty adapting to increases in afterload. Generally, the progression of pulmonary hypertension is slow, allowing the right ventricle to adapt to the increases. Infectious exacerbations and exercise can increase the pulmonary pressures significantly, worsening left ventricular filling via interventricular dependence. Patients with LVDD and elevated LV filling pressures cause an increase in pulmonary post-capillary pressures [49].We found RVEDD was significantly greater in the COPD group, thus the hearts of some patients with COPD undergo conformational changes. This finding helps illustrate the potential link between right ventricular dilation and left ventricular diastolic dysfunction.

The reverse Bernheim phenomenon may explain the influence of the right ventricular volume and or pressure on the left ventricular function $[30,50]$. The interdependence of the ventricles is due to the left and right chambers being different from one another in sustainable pressures, strength, elasticity and size and yet anatomically bound to one another by the pericardium and their shared interventricular septum. It is postulated that pericardial restraint ensures that with increased pulmonary artery / right ventricular pressures as may

occur with COPD, the interventricular septum will deviate into the left ventricle. As a result, there is flattening or reversal of the septal curvature and compression of the left ventricle at end-systole, which may persist in the early diastolic filling period [24,30,49-51]. Our findings indicate that in COPD, LV filling is reduced during early diastole-as indicated by the lower Epv-when septal geometry is compromised, and there is a compensatory increase in late diastolic filling, as indicated by the increased Apv and inverted E/A ratio.

Other factors may contribute to LVDD such as diabetes mellitus, coronary artery disease, hypertension, obesity, and age. The strengthof our study is the analysis of age, sex and co-morbidity matched studies, suggesting COPD as a risk factor for LVDD.

\section{Clinical Implications}

Worsening dyspnea in patients with COPD is often thought to be due to progression of the lung disease, however LVDD may lead to increased pulmonary post-capillary pressures and clinically significant HFpEF $[30,49]$. Our study underscores that COPD patients may have a higher prevalence of LVDD and thus may be more sensitive to preload changes and elevations in pulmonary artery pressure, which may occur from various causes such as arrhythmias and infectious exacerbations [30].

COPD is linked to cardiovascular disease, particularly heart failure [34]. This review underscores the increased risk of LVDD in this group of patients, which may be present even in the early stages of the COPD and often times may go undiagnosed. Fontes-Carvalho showed that COPD patients with LVDD are at increased risk for hospitalization for exacerbation [37]. Reversible ischemic defects may be present at a higher rate in patients with advanced COPD and LVDD thus vigilance for significant coronary disease is prudent $[21,52]$.

Further studies to explore diastolic stress testing in COPD patients may be interesting. A study in non-COPD patents demonstrated that $\mathrm{E} / \mathrm{e}^{\prime}>13$ with exercise has incremental prognostic power for ischemic and heart failure events in follow up [53]. Whether similar findings in patients with COPD are seen merits study. Further research highlighting the prevalence of RV dysfunction in COPD patients with LVDD, both on morbidity and mortality, is needed. It is possible that studying left ventricular lusitropy in COPD patients may provide diagnostic and prognostic information, and perhaps help direct therapy.

\section{Funding}

This work is supported, in part, by the efforts of Dr. Moro O. Salifu M.D., M.P.H., M.B.A., M.A.C.P., Professor and Chairman of Medicine through NIH Grant number S21MD012474.

\section{Competing Interests}

The authors declare that they have no competing interests.

\section{References}

1. Divo M, Cote C, de Torres JP, Casanova C, Marin JM, et al. (2012) Comorbidities and risk of mortality in patients with chronic obstructive pulmonary disease. Am J Respir Crit Care Med 186: 155-161.

2. Heidenreich PA, Albert NM, Allen LA, Bluemke DA, Butler J, et al, (2013) Forecasting the impact of heart failure in the United States: a policy statement from the American Heart Association. Circ Heart Fail 6: 606-619. 
Citation: Zhyvotovska A, Yusupov D, Kamran H, Al-Bermani T, Abdul R, et al. (2019) Diastolic Dysfunction in Patients with Chronic Obstructive Pulmonary Disease: A Meta-Analysis of Case Controlled Studies. Int J Clin Res Trials 4: 137. doi: https://doi.org/10.15344/2456-8007/2019/137

Page 10 of 11

3. Cavaillès A, Brinchault-Rabin G, Dixmier A, Goupil F, Gut-Gobert C, et al. (2013) Comorbidities of COPD. Eur Respir Rev 22: 454-475.

4. Clini EM, Boschetto P, Lainscak M, Janssens W (2014) Comorbidities in chronic obstructive pulmonary disease from assessment to treatment. Biomed Res Int 2014: 414928

5. Ponikowski P, Voors AA, Anker SD, Bueno H, Cleland JG, et al. (2016) 2016 ESC Guidelines for the Diagnosis and Treatment of Acute and Chronic Heart Failure. Rev Esp Cardiol 69: 1167.

6. Nagueh SF, Smiseth OA, Appleton CP, Byrd BF 3rd, Dokainish H, et al. (2009) Recommendations for the evaluation of left ventricular diastolic function by echocardiography. Eur J Echocardiogr 10: 165-193.

7. Owan TE, Hodge DO, Herges RM, Jacobsen SJ, Roger VL, et al. (2006) Trends in prevalence and outcome of heart failure with preserved ejection fraction. N Engl J Med 355: 251-259.

8. Fontes-Carvalho $R$, Ladeiras-Lopes $R$, Bettencourt $P$, Leite-Moreira $A$ Azevedo A, et al. (2015) Diastolic dysfunction in the diabetic continuum: association with insulin resistance, metabolic syndrome and type 2 diabetes. Cardiovasc Diabetol 14: 4

9. Aslam F, Bandeali SJ, Khan NA, Alam M (2013) Diastolic dysfunction in rheumatoid arthritis: a meta-analysis and systematic review. Arthritis Care Res (Hoboken) 65: 534-543.

10. Redfield MM (2016) Heart Failure with Preserved Ejection Fraction. N Eng J Med 375: 1868-1877.

11. Halley CM, Houghtaling PL, Khalil MK, Thomas JD, Jaber WA, et al. (2011) Mortality rate in patients with diastolic dysfunction and normal systolic function. Arch Intern Med 171: 1082-1087.

12. Redfield MM, Jacobsen SJ, Burnett JC, Jr., Mahoney DW, Bailey KR, et al. (2003) Burden of systolic and diastolic ventricular dysfunction in the community: appreciating the scope of the heart failure epidemic. JAMA 289: 194-202.

13. Ahmed A, Aronow WS, Fleg JL (2006) Higher New York Heart Association classes and increased mortality and hospitalization in patients with heart failure and preserved left ventricular function. Am Heart J 151: 444-450.

14. Jones RC, Francis GS, Lauer MS (2004) Predictors of mortality in patients with heart failure and preserved systolic function in the Digitalis Investigation Group trial. J Am Coll Cardiol 44: 1025-1029.

15. Persson $\mathrm{H}$, Lonn $\mathrm{E}$, Edner $\mathrm{M}$, Baruch $\mathrm{L}$, Lang CC, et al. (2007) Diastolic dysfunction in heart failure with preserved systolic function: need for objective evidence: results from the CHARM Echocardiographic SubstudyCHARMES. J Am Coll Cardiol 49: 687-694.

16. Felker GM, Allen LA, Pocock SJ, et al. Red cell distribution width as a nove prognostic marker in heart failure: data from the CHARM Program and the Duke Databank. J Am Coll Cardiol 50: 40-47.

17. Nagueh SF, Smiseth $\mathrm{OA}$, Appleton $\mathrm{CP}$, Byrd BF 3rd, Dokainish $\mathrm{H}$, et al (2016) Recommendations for the Evaluation of Left Ventricular Diastolic Function by Echocardiography: An Update from the American Society of Echocardiography and the European Association of Cardiovascular Imaging. Eur Heart J Cardiovasc Imaging 17: 1321-1360.

18. AboEl-Magd Gehan HT, Aly Mohamed H., Mabrouk Maaly M (2017) Echocardiography and $\mathrm{N}$-terminal-pro-brain natriuretic peptide in assessment of left ventricualr diastolic dysfunction in stable COPD in relation to disease severity. Egyptian Journal of Chest Diseases and Tuberculosis 66: 75-80

19. Acharya V RS, Seshaderi S, Shetty R (2018) Assessment of Diastolic Dysfunction Parameters and Cardiac Chamber Size in Smokers with COPD: A Case Control Study. Journal of Clinical and Diagnostic Research.

20. Boussuges A, Pinet $C$, Molenat F, Burnet $H$, Ambrosi P, et al. (2000) Left atrial and ventricular filling in chronic obstructive pulmonary disease. An echocardiographic and Doppler study. Am J Respir Crit Care Med 162: 670675.

21. Eweda I, Hamada G (2016) Concordance between Doppler and pulsedwave Doppler tissue imaging in estimation of the degree of left ventricular dysfunction and correlating it to the degree of chronic obstructive pulmonary disease. J Saudi Heart Assoc 28: 15-21.

22. Faludi R, Hajdu M, Vertes V, et al. (2016) Diastolic Dysfunction Is a Contributing Factor to Exercise Intolerance in COPD. COPD 13: 345-351.

23. Farouk $\mathrm{H}$, Albasmi M, El Chilali $\mathrm{K}$, et al. Left ventricular diastolic dysfunction in patients with chronic obstructive pulmonary disease: Impact of methods of assessment. Echocardiography 34: 359-364.
24. Funk GC, Lang I, Schenk P, Valipour A, Hartl S, et al. (2008) Left ventricular diastolic dysfunction in patients with COPD in the presence and absence of elevated pulmonary arterial pressure. Chest 133: 1354-1359.

25. Huang YS, Feng YC, Zhang J, Bai L, Huang W, et al. (2015) Impact of chronic obstructive pulmonary diseases on left ventricular diastolic function in hospitalized elderly patients. Clin Interv Aging 10: 81-87.

26. Kubota Y, Asai K, Murai K, Tsukada YT, Hayashi H, et al. (2016) COPD advances in left ventricular diastolic dysfunction. Int J Chron Obstruct Pulmon Dis 11: 649-655.

27. Malerba M, Ragnoli B, Salameh M, Sennino G, Sorlini ML, et al. (2011) Sub-clinical left ventricular diastolic dysfunction in early stage of chronic obstructive pulmonary disease. J Biol Regul Homeost Agents 25: 443-451.

28. Pelà G, Li Calzi M, Pinelli S, Andreoli R, Sverzellati N, et al. Left ventricular structure and remodeling in patients with COPD. Int J Chron Obstruct Pulmon Dis 11: 1015-1022.

29. Sabit R, Bolton CE, Fraser AG, Edwards JM, Edwards PH, et al. (2010) Subclinical left and right ventricular dysfunction in patients with COPD. Respir Med 104: 1171-1178.

30. Suchoń E, Tracz W, Podolec P, Pieculewicz M, Płazak W, et al. (2007) [Evaluation of left ventricular function in patients with chronic obstructive pulmonary disease]. Pol Arch Med Wewn 117: 26-30.

31. El Wahsh RAA, Ahmed MK, Yassen RI (2013) Evaluation of Left ventricular function in patients with chronic obstructive pulmonary diease with or without pulmonary hypertension. Egyptian Journal of Chest Diseases and Tuberculosis 62: 575-582.

32. Yilmaz R, Gencer M, Ceylan E, Demirbag R (2005) Impact of chronic obstructive pulmonary disease with pulmonary hypertension on both left ventricular systolic and diastolic performance. J Am Soc Echocardiogr 18: 873-881.

33. Sin DD, Man SF (2005) Chronic obstructive pulmonary disease as a risk factor for cardiovascular morbidity and mortality. Proc Am Thorac Soc 2 . 8-11.

34. Finkelstein J, Cha E, Scharf SM (2009) Chronic obstructive pulmonary disease as an independent risk factor for cardiovascular morbidity. Int J Chron Obstruct Pulmon Dis 4: 337-349.

35. Gladden JD, Linke WA, Redfield MM (2014) Heart failure with preserved ejection fraction. Pflugers Arch 466: 1037-1053.

36. LeWinter MM, Meyer M (2013) Mechanisms of diastolic dysfunction in heart failure with a preserved ejection fraction: If it's not one thing it's another. Circ Heart Fail 6: 1112-1115.

37. Fontes-Carvalho R, Leite-Moreira A (2011) Heart failure with preserved ejection fraction: fighting misconceptions for a new approach. Arq Bras Cardiol 96: 504-514.

38. Biering-Sorensen T, Mogelvang R, Jensen JS (2015) Prognostic value of cardiac time intervals measured by tissue Doppler imaging $\mathrm{M}$-mode in the general population. Heart 101: 954-960.

39. Chhabra SK, De S (2005) Cardiovascular autonomic neuropathy in chronic obstructive pulmonary disease. Respir Med 99: 126-133.

40. Barr RG, Bluemke DA, Ahmed FS, Carr JJ, Enright PL, et al. (2010) Percent emphysema, airflow obstruction, and impaired left ventricular filling. $\mathrm{N}$ Engl J Med 362: 217-227.

41. Watz H, Waschki B, Meyer T, Kretschmar G, Kirsten A, et al. (2010) Decreasing cardiac chamber sizes and associated heart dysfunction in COPD: role of hyperinflation. Chest 138: 32-38.

42. Jorgensen K, Muller MF, Nel J, Upton RN, Houltz E, et al. (2007) Reduced intrathoracic blood volume and left and right ventricular dimensions in patients with severe emphysema: an MRI study. Chest 131: 1050-1057.

43. Held M, Jany BH (2013) Pulmonary hypertension in COPD. Respir Care 58: 86-91.

44. Barbera JA, Peinado VI, Santos S (2003) Pulmonary hypertension in chronic obstructive pulmonary disease. Eur Respir J 21: 892-905.

45. Chaouat A, Naeije R, Weitzenblum E (2008) Pulmonary hypertension in COPD. Eur Respir J 32: 1371-1385.

46. Cargill RI, Kiely DG, Lipworth BJ (1995) Adverse effects of hypoxaemia on diastolic filling in humans. Clin Sci 89: 165-169.

47. Paulus WJ, Tschope C (2013) A novel paradigm for heart failure with preserved ejection fraction: comorbidities drive myocardial dysfunction and remodeling through coronary microvascular endothelial inflammation. J Am Coll Cardiol 62: 263-271. 
Citation: Zhyvotovska A, Yusupov D, Kamran H, Al-Bermani T, Abdul R, et al. (2019) Diastolic Dysfunction in Patients with Chronic Obstructive Pulmonary Disease: A Meta-Analysis of Case Controlled Studies. Int J Clin Res Trials 4: 137. doi: https://doi.org/10.15344/2456-8007/2019/137

Page 11 of 11

48. Laurent $S$, Cockcroft J, Van Bortel L, Boutouyrie P, Giannattasio C, et al. (2006) Expert consensus document on arterial stiffness: methodological issues and clinical applications. Eur Heart J 27: 2588-2605.

49. Moustapha A, Kaushik V, Diaz S, Kang SH, Barasch E, et al. (2001) Echocardiographic evaluation of left-ventricular diastolic function in patients with chronic pulmonary hypertension. Cardiology 95: 96-100.

50. Alpert JS (2001) The effect of right ventricular dysfunction on left ventricular form and function. Chest 119: 1632-1633.

51. Tutar E, Kaya A, Güleç S, Ertaş F, Erol C, et al. (1999) Echocardiographic evaluation of left ventricular diastolic function in chronic cor pulmonale. Am J Cardiol 83: 1414-1417.

52. Bhattacharyya P, Acharjee D, Ray SN, Sharma RK, Tiwari P, et al. (2012) Left ventricular diastolic dysfunction in COPD may manifest myocardial ischemia. COPD 9: 305-309.

53. Holland DJ, Prasad SB, Marwick TH (2010) Prognostic implications of left ventricular filling pressure with exercise. Circ Cardiovasc Imaging 3: 149156 